\title{
Relato de caso de tratamento endovascular de fístula aorto-entérica secundária
}

\author{
A case report of an endovascular treatment of secondary aortenteric fistula \\ Fabio Henrique Rossi', Nilo Mitsuru Izukawa², Akash Kuzhiparambil Prakasan³, Heraldo Antônio Barbato³, \\ Antonio Kambara ${ }^{4}$, Patrik Bastos Metzger ${ }^{5}$, Frederico Augusto de Carvalho Linhares Filho ${ }^{5}$, Camila Baumann Betelli ${ }^{5}$
}

\begin{abstract}
Resumo
O tratamento tradicional da fístula aorto-entérica secundária baseia-se na retirada cirúrgica da prótese, desbridamento aórtico, enxerto extra-anatômico, ou in situ, nos casos em que o campo cirúrgico apresenta-se sem sinais de infecção. Recentemente, alguns autores vêm preconizando o tratamento endovascular em pacientes instáveis e com alto risco cirúrgico. Apresentamos um relato de caso de paciente portador de fístula aorto-entérica secundária tratado inicialmente por via endovascular.
\end{abstract}

Palavras-chave: aneurisma; enxerto vascular; fístula do sistema digestório; procedimentos endovasculares.

\begin{abstract}
Traditional treatment of secondary aortoenteric fistula is based on open surgery followed by device removal, aortic debridement and extra-anatomic or in situ by-pass when no signs of local infection are found. Recently, some authors have been advocating endovascular treatment in unstable and high-risk patients. We present a case report of a patient who underwent initial endovascular treatment of a secondary aortoenteric fistula.
\end{abstract}

Keywords: aneurysm; vascular grafting; digestive system fistula; endovascular procedures.

Introdução

A fístula aorto-entérica secundária representa uma situação rara, mas extremamente grave, que pode ocorrer após a cirurgia aórtica. Ocorre em 0,3 a 2,5\% dos casos cirúrgicos e menos frequentemente após o tratamento endovascular. É definida como uma comunicação anômala entre a prótese e o tubo digestório, podendo ocorrer em qualquer um de seus segmentos, sendo mais comum com o duodeno. Sua apresentação clínica envolve a hemorragia gastrointestinal em $70 \%$, sepsis em $16 \%$ ou ambas as condições em $14 \%$ dos $\operatorname{casos}^{1,2}$.
Os métodos diagnósticos mais utilizados são a tomografia computadorizada, a esofagoduodenogastroendoscopia e a arteriografia. Apesar do domínio das técnicas cirúrgicas utilizadas, um grande número de pacientes evolui para o óbito (35-75\%) $)^{3}$. Dessa forma, o tratamento endovascular surgiu como alternativa, sobretudo nos pacientes que se apresentam com instabilidade hemodinâmica. O objetivo do nosso estudo foi relatar um caso de fístula aorto-entérica secundária tratado em nosso serviço e realizar uma revisão da restrita literatura comparativa entre os tratamentos cirúrgicos convencional e endovascular.

Trabalho realizado no Instituto Dante Pazzanese de Cardiologia - São Paulo (SP), Brasil.

'Doutor em Medicina pela Universidade de São Paulo (USP) - São Paulo (SP), Brasil. Médico assistente da seção médica de Cirurgia Vascular e membro do Centro de Intervenções Endovasculares (CIEV) do Instituto Dante Pazzanese de Cardiologia - São Paulo (SP), Brasil.

²Doutor em medicina pela USP - São Paulo (SP), Brasil. Chefe da seção médica de Cirurgia Vascular e do CIEV do Instituto Dante Pazzanese de Cardiologia - São Paulo (SP), Brasil.

${ }^{3}$ Médico assistente da seção médica de Cirurgia Vascular e membro do CIEV do Instituto Dante Pazzanese de Cardiologia - São Paulo (SP), Brasil.

${ }^{4}$ Mestre em Medicina pela Universidade Federal de São Paulo (UNIFESP) - São Paulo (SP), Brasil; Chefe da seção médica de Radiologia e do CIEV do Instituto Dante Pazzanese de Cardiologia - São Paulo (SP), Brasil; Membro titular do Colégio Brasileiro de Radiologia - São Paulo (SP), Brasil.

5Médico aprimorando do CIEV do Instituto Dante Pazzanese de Cardiologia - São Paulo (SP), Brasil.

Conflito de interesse: nada a declarar

Fonte de financiamento: nenhuma

Submetido em: 25.01.12. Aceito em: 21.06.12

J Vasc Bras. 2012;11(3):236-239. 


\section{Relato de caso}

Paciente do sexo masculino, 66 anos de idade, hígido e muito ativo, manifestava história de fezes sanguinolentas e hematoquesia havia uma semana. Apresentava história pregressa de enxerto aorto-bi-ilíaco com prótese de Dacron por aneurisma de aorta abdominal há 11 anos. Encontrava-se em preparação para correção endovascular de pseudoaneurisma localizado em boca anastomótica ilíaca esquerda. Não referia demais queixas vasculares. Tinha como antecedentes hipertensão arterial, diabetes mellitus, dislipidemia e ex-tabagismo. Ao exame físico, apresentava-se em bom estado geral, levemente hipocorado e afebril. Demonstrava massa abdominal palpável e dolorosa, sem sinais de peritonite. Ao exame vascular, apresentava todos os pulsos palpáveis, exceto os pós-poplíteos que se apresentavam diminuídos em sua amplitude. Os exames laboratoriais revelaram hemoglobina de $9,8 \mathrm{~g} / 100 \mathrm{~mL}$ e hematócrito de $28 \%$. Apresentava leucocitose de $12.000 / \mathrm{mm}^{3}$, sem desvio à esquerda. $\mathrm{O}$ coagulograma, ureia, creatinina, sódio, potássio e enzimas hepáticas eram normais.

O paciente foi submetido à nova angiotomografia, de urgência, com fase arterial e venosa quando foi evidenciada a presença de, além do volumoso pseudoaneurisma, já demonstrado na anastomose ilíaca esquerda, imagem sugestiva de sangramento e infecção retossigmoideana (Figura 1). Logo após o exame, o paciente apresentou sangramento profuso e grave queda da pressão arterial. Nossa primeira hipótese diagnóstica foi de ruptura do pseudoaneurisma; como se apresentava em condições clínicas desfavoráveis para a cirurgia convencional, o paciente foi

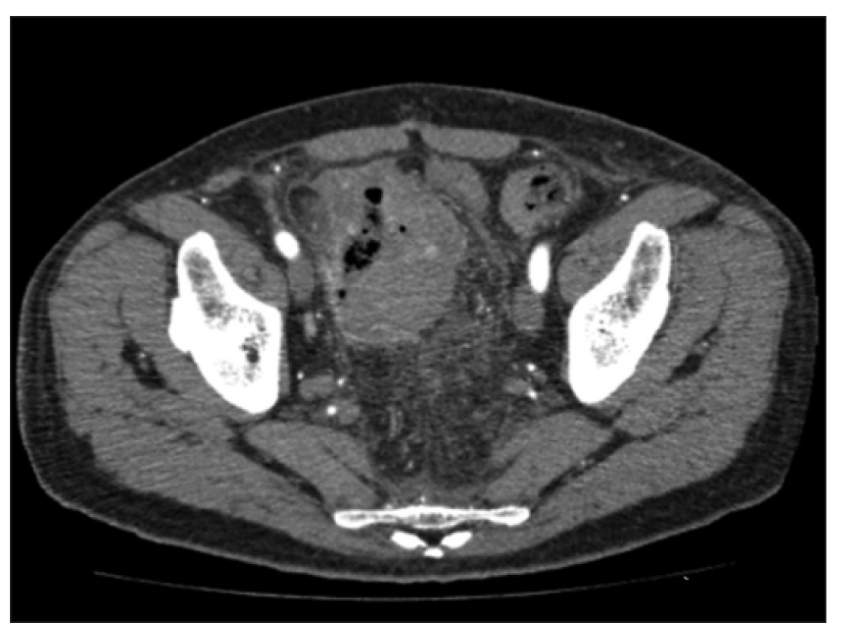

Figura 1. Angiotomografia demonstrando volumoso pseudoaneurisma em topografia de anastomose ilíaca esquerda apresentando imagem sugestiva de sangramento e infecção. encaminhado imediatamente para a realização de tratamento endovascular. A infecção e a lesão sigmoideana seriam tratadas em um segundo tempo, com o paciente em condições mais estáveis.

O paciente foi submetido à anestesia geral e mantido em hipotensão permissiva. As duas femorais foram imediatamente dissecadas e puncionadas sobre visualização direta. Foi então implantada endoprótese Gore Excluder $23 \times 120 \times 10$ sem dificuldades técnicas, uma vez que a anatomia apresentou-se favorável para a realização do procedimento. (Figura 2).

$\mathrm{O}$ paciente permaneceu na unidade de terapia intensiva por 72 horas. Após uma semana de observação, já na enfermaria, período em que permaneceu estável clínico-laboratorialmente, o paciente apresentou novo sangramento, dessa vez em pequena quantidade e sem repercussão hemodinâmica. Optamos pela realização de laparotomia exploradora imediata, pois, em nossa opinião, esse novo episódio de sangramento, associado com os sinais radiológico de infecção, colocava o paciente sob risco de novo sangramento, profuso e fatal. Durante o ato operatório, foi verificada a presença de contaminação grosseira por fezes no local do pseudoaneurisma e fístula entérica. Optou-se pela realização de ligadura da aorta e artérias ilíacas, retirada das próteses cirúrgica e endovascular, desbridamento amplo do tecido necrótico e infectado, e lavagem da cavidade abdominal. Foi ainda realizada colostomia em alça para permitir posterior correção e enxerto axilo bifemoral. O paciente evoluiu com instabilidade hemodinâmica refratária às medidas clínicas adotadas e óbito no primeiro dia de pós-operatório.

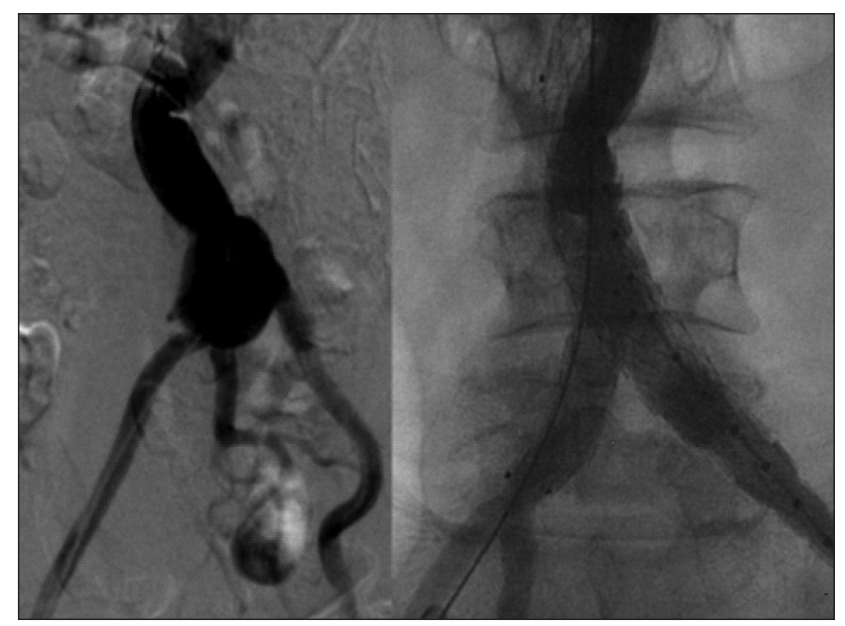

Figura 2. Angiografia final demonstrando implante de uma endoprótese bifurcada Gore Excluder $23 \times 120 \times 10$ mm sem sinal de vazamentos. 


\section{Discussão}

$\mathrm{Na}$ atualidade, a grande maioria dos pacientes acometidos por fístulas aorto-entéricas secundárias é tratada por meio da retirada cirúrgica imediata da prótese, ou endoprótese, desbridamento do material necrótico e infeccioso, ligadura e sutura aórtica, e realização de enxerto extra-anatômico, ou in situ, com prótese impregnada em rifampicina, ou menos comumente com veia autógena do sistema venoso profundo. Esses procedimentos prolongados são associados a altíssimos índices de morbimortalidade ${ }^{1,2}$.

O tratamento endovascular tem se demonstrado uma alternativa terapêutica viável, associada a menor trauma e tempo cirúrgico, menor necessidade de transfusão sanguínea e menores índices de morbimortalidade. Entretanto, essa conduta é raramente realizada, pouco descrita, e não existem estudos randomizados e séries controladas que verificaram o real benefício clínico desse tipo de conduta ${ }^{3}$.

No caso clínico aqui apresentado, apesar da estabilidade inicial, o paciente evolui com choque hemorrágico durante a internação, o qual foi prontamente revertido com o implante emergencial de uma endoprótese bifurcada, que interrompeu o sangramento e permitiu a estabilização hemodinâmica. Essa modalidade de tratamento envolve menores perdas sanguíneas e, possivelmente, foi importante para o sucesso terapêutico inicial nesse paciente. Na segunda cirurgia, foi verificada a presença de infecção grosseira das próteses, motivo pelo qual foi necessária a retirada delas e execução de cirurgia complexa e demorada. Alguns autores, no caso de ausência de sepsis, preconizam o tratamento endovascular associado à antibioticoterapia prolongada e seguimento clínico $^{3,4}$, o que não foi possível em nosso caso em razão da presença de infecção grosseira. A reconstrução do trato intestinal, a limpeza e o desbridamento cirúrgico do tecido necrótico parecem melhorar os resultados ${ }^{5,6}$.

Dados da literatura apontam que, apesar dos favoráveis resultados iniciais obtidos com o tratamento endovascular, pode haver recidiva da infecção e do sangramento. Essa evolução desfavorável parece acometer sobretudo aqueles pacientes portadores de sepsis em sua apresentação inicial ${ }^{7,8}$. Em nosso caso, embora não haja sepsis como apresentação inicial, durante a laparotomia exploradora, realizada após o segundo episódio de sangramento, pode ser verificada a presença de infecção grosseira, inclusive com a presença de conteúdo fecal.

Kakkos et al.8 recentemente publicaram uma metanálise sobre os resultados obtidos com o tratamento endovascular: em 59 casos descritos na literatura, verificaram sucesso técnico em $93 \%$ e mortalidade após 30 dias de acompanhamento de 8,5\%. Ao seguimento tardio, 10 (19\%) pacientes evoluíram com recidiva de sangramento. Dezessete (32\%) pacientes desenvolveram sepsis, e foram tratados clinicamente em 8 casos (7 com sucesso). Os pacientes que tiveram reconstrução do trato intestinal evoluíram com menores índices de recidiva $(\mathrm{p}=0,022)$. A mortalidade após 12 e 24 meses de acompanhamento foi $15 \%$ e $19 \%$, respectivamente, significantemente pior quando houve recidiva de sangramento $(\mathrm{p}=0,001)$. $O$ prognóstico foi pior na presença de sepsis pré-operatória, fístula com o intestino grosso, correção cirúrgica convencional, não correção cirúrgica do tubo digestório e recorrência da fístula. Os autores concluem que o tratamento endovascular apresenta resultados aceitáveis a curto e médio prazo e, na atualidade, mortalidade menor do que aquela obtida com o tratamento cirúrgico convencional'.

Existem poucos estudos, não há definições e não há consenso sobre quais pacientes devem ser submetidos aos dois métodos de tratamento. A hemorragia ocasionada pela fístula aorto-entérica, associada a quadro séptico ou não, é sempre um evento catastrófico, e em pacientes instáveis o procedimento cirúrgico convencional está associado a altíssimos índices de mortalidade.

Concluímos que o tratamento endovascular da fístula aorto-entérica secundária pode representar uma alternativa inicial ao tratamento cirúrgico convencional em pacientes sem sinais clínicos e operatórios de sepsis e infecção grave, sobretudo em pacientes instáveis e com alto risco cirúrgico.

\section{Referências}

1. Kuestner LM, Reilly LM, Jicha DL, Ehrenfeld WK, Goldstone J, Stoney RJ. Secondary aortoenteric fistula: contemporary outcome with use of extraanatomic bypass and infected graft excision. I Vasc Surg. 1995;21:184-95; discussion 195-6. PMid:7853593.

2. Sharif MA, Lee B, Lau LL, et al. Prosthetic stent graft infection after endovascular abdominal aortic aneurysm repair.J Vasc Surg. 2007;46:4428. PMid:17826231. http://dx.doi.org/10.1016/j.jvs.2007.05.027

3. Burks JA, Faries PL, Gravereaux EC, Hollier LH, Marin ML. Endovascular repair of bleeding aortoenteric fistulas: a 5-year experience. J Vasc Surg. 2001;34:1055-9. PMid:11743560. http://dx.doi.org/10.1067/ mva.2001.119752

4. Baril DT, Carroccio A, Ellozy SH, et al. Evolving strategies for the treatment of aortoenteric fistulas. J Vasc Surg. 2006;44:250-7. http://dx.doi. org/10.1016/j.jvs.2006.04.031

5. Laganà D, Carrafiello G, Mangini M, et al. Endovascular treatment of anastomotic pseudoaneurysms after aorto-iliac surgical reconstruction. Cardiovasc Intervent Radiol. 2007;30:1185-91. http://dx.doi. org/10.1007/s00270-007-9047-0 
6. Brountzos EN, Vasdekis S, Kostopanagiotou G, et al. Endovascular treatment of a bleeding secondary aorto-enteric fistula. A case report with 1-year follow-up. Cardiovasc Intervent Radiol. 2007;30:1037-41. PMid:17546398. http://dx.doi.org/10.1007/s00270-007-9099-1

7. Antoniou GA, Koutsias S, Antoniou SA, Georgiakakis A, Lazarides MK, Giannoukas AD. Outcome after endovascular stent graft repair of aortoenteric fistula: A systematic review. J Vasc Surg. 2009;49:782-9. PMid:19028054. http://dx.doi.org/10.1016/j.jvs.2008.08.068

8. Kakkos SK, Antoniadis PN, Klonaris CN, et al. Open or endovascular repair of aortoenteric fistulas? A multicentre comparative study. Eur J Vasc Endovasc Surg. 2011;41:625-34. PMid:21324718. http://dx.doi. org/10.1016/j.ejvs.2010.12.026

9. Kakkos SK, Papadoulas S, Tsolakis IA. Endovascular management of arterioenteric fistulas: a systemic review and meta-analysis of the literature. J Endovasc Ther. 2011;18:66-77. PMid:21314352. http://dx.doi. org/10.1583/10-3229.1

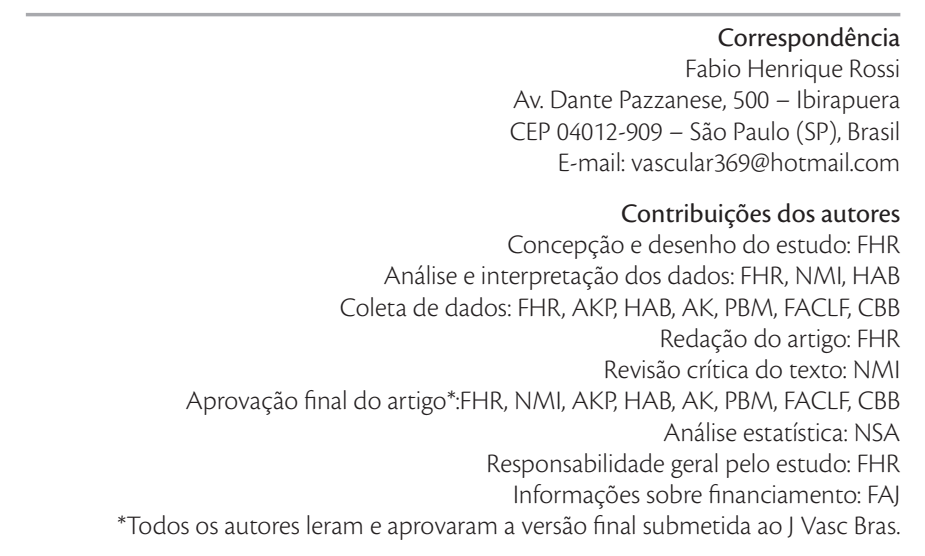

Correspondência Dante Pazzanese, 500 - Ibirapuera 04012-909 - São Paulo (SP), Brasil

Contribuições dos autores Concepção e desenho do estudo: FHR dados: FHR, NMI, HAB Redação do artigo: FHR
Re isão crítica do texto: NMI *Todos os autores leram e aprovaram a versão final submetida ao J Vasc Bras. 